\title{
Does Long-term Experimental Antiorthostasis Lead to Cardiovascular Deconditioning in the Rat?
}

\author{
G. RAFFAI, C. CSEKÖ ${ }^{1}$, L. KOCSIS, L. DÉZSI ${ }^{2}$, E. MONOS \\ Institute of Human Physiology and Clinical Experimental Research, Faculty of Medicine, \\ Semmelweis University, and ${ }^{1}$ Department of General Pharmacology, and ${ }^{2}$ Department of \\ Pharmacology and Drug Safety Research of Gedeon Richter Plc., Budapest, Hungary
}

Received April 10, 2007

Accepted November 5, 2008

On-line January 17, 2008

\begin{abstract}
Summary
Microgravity or simulated microgravity induces acute and chronic cardiovascular responses, whose mechanism is pivotal for understanding of physiological adaptation and pathophysiological consequences. We investigated hemodynamic responses of conscious Wistar rats to $45^{\circ}$ head-down tilt (HDT) for 7 days. Arterial blood pressure (BP) was recorded by telemetry. Heart rate $(H R)$, spectral properties and the spontaneous baroreflex sensitivity (sBRS) were calculated. Head-up tilt (HUT) was applied for $2 \mathrm{~h}$ before and after HDT to assess the degree of any possible cardiovascular deconditioning. Horizontal control BP and HR were $112.5 \pm 2.8 \mathrm{mmHg}$ and $344.7 \pm 10 \mathrm{bpm}$, respectively. HDT elicited an elevation in BP and HR by $8.3 \%$ and $8.8 \%$, respectively, in less than $1 \mathrm{~h}$. These elevations in BP and HR were maintained for 2 and 3 days, respectively, and then normalized. Heart rate variability was unchanged, while sBRS was permanently reduced from the beginning of HDT $(1.01 \pm 0.08$ vs. $0.74 \pm 0.05 \mathrm{~ms} / \mathrm{mmHg}$ ). HUT tests before and after HDT resulted in BP elevations (6.9 vs. $11.6 \%$ ) and SBRS reduction ( 0.44 vs. $0.37 \mathrm{~ms} / \mathrm{mmHg}$ ), respectively. The pressor response during the post-HDT HUT test was accompanied by tachycardia (13.7 \%). In conclusion, chronic HDT does not lead to symptoms of cardiovascular deconditioning. However the depressed SBRS and tachycardic response seen during the post-HDT HUT test may indicate disturbances in cardiovascular control.
\end{abstract}

\section{Key words}

Antiorthostasis • Baroreflex sensitivity • Cardiovascular deconditioning • Microgravity

\section{Corresponding author}

Gábor Raffai MSc, PhD., Institute of Human Physiology and Clinical Experimental Research, Semmelweis University, Budapest, Hungary, Üllői út 78/a, H-1082, Fax.: (36-1)334-3162. E-mail: raffai@elet2.sote.hu

\section{Introduction}

Microgravity and simulated microgravity induce several acute and chronic adaptive responses. The longterm responses include cephalad fluid shifts, a decrease in plasma volume, reduced capacity for physical work, atrophy of weight bearing muscles, loss of bone calcium, cardiovascular deconditioning (for reviews see Convertino 1999, 2002a,b, Zhang 2001, Zhang et al. 2001), a decrease in periadventitial innervation density of arteries, and a reduced myogenic response of veins in the extremities (Zhang 2001, Monos et al. 2007).

Cardiovascular deconditioning may affect both myocardial function (heart rate, stroke volume, cardiac output, left ventricular end diastolic volume, cardiac contractility, ejection fraction) and vascular function (peripheral vascular resistance and capacitance, blood pressure, regional blood flow, vasoconstrictor reserve and responsiveness) (Somody et al. 1998, Convertino 1999, 2002a,b, Zhang 2001, Zhang et al. 2001, Monos et al. 2007). Cardiac vagal nerve activity, occurring in response to carotid baroreceptor stimulation was also reported to be attenuated in head-down tilt bedrest and in spaceflight studies (Convertino 1999). Decreased blood volume, increased venous capacitance, reduced vascular flow resistance, and impairment of baroreflex sensitivity may lead to an inability to maintain arterial blood pressure in the erect body position, resulting in orthostatic intolerance following either spaceflight or chronic bedrest (Convertino 1999, 2002a,b, Zhang 2001, Zhang et al. 2001, Monos et al. 2007).

Thus, the long-term physiological consequences 
of microgravity, and simulated microgravity have been intensively studied in both human subjects and animal models (Musacchia and Fagette 1997, Convertino 1999, 2002a,b, Zhang 2001, Zhang et al. 2001, Monos et al. 2007). However, the mechanism of physiological responses leading to cardiovascular deconditioning has not been analyzed yet in depth. In addition, published data regarding microgravitational adaptation are controversial. As noted by Musacchia and Fagette (1997), cardiovascular responses in the rat models are divergent in different laboratories, probably due to variations of protocols and differences in the applied techniques (chronic catheterization, anesthesia). For example, blood pressure measured by chronic catheterization was reported to be elevated from the first day of head-down tilting made by tail suspension in the rat (Musacchia et al. 1992), while no change was observed by others (Brizzee and Walker 1990, Fagette et al. 1995, Somody et al. 1998, Bayorh et al. 1999, 2001, Socci et al. 2000, Tarasova et al. 2001, Eatman et al. 2003). Recently, we found that conscious telemetered rats exposed to either $45^{\circ}$ head-up tilt (HUT) or head-down tilt (HDT) for 120 min in long tubular cages responded with a sustained elevation of arterial blood pressure via sympathetic activation (Raffai et al. 2005, 2006).

Therefore, based on our previous observations (Lóránt et al. 2005, Raffai et al. 2005, 2006) the present study investigated blood pressure and heart rate responses, their spectral properties, and the spontaneous baroreflex sensitivity using cardiovascular telemetry (Brockway et al. 1991) in conscious Wistar rats subjected to our chronic head-down tilt model (Lóránt et al. 2005). In addition, head-up tilts (HUT) were applied for $2 \mathrm{~h}$ preceding and following HDT period, in order to assess the degree of a possible cardiovascular deconditioning. The specific aims of the experiments were to: 1) examine the influence of 7-day HDT on body temperature, somatomotor and feeding activity, 2) to characterize the changes of arterial blood pressure, heart rate, autonomic nervous activity and baroreflex sensitivity during chronic antiorthostasis, and 3) to compare orthostatic cardiovascular responses to HUT before and after HDT.

\section{Methods}

\section{Experimental animals}

Male adult Wistar rats ( $\mathrm{n}=8$, Harlan, Germany) weighing 300-350 g were used for the experiments. All animals were housed individually with $12 \mathrm{~h}$ light and dark cycles (07:00-19:00, 19:00-07:00 h, respectively) at $20-22{ }^{\circ} \mathrm{C}$. They had free access to standard laboratory rat chow and tap water. The investigation conforms with the Guide for the Care and Use of Laboratory animals published by the US National Institutes of Health (NIH Publication No. 85-23, revised 1996). Studies were carried out according to the guidelines of Hungarian law on animal protection (243/1998) and approved by the Semmelweis University Committee on the Ethical Use of Experimental Animals (590/99 Rh).

\section{Surgery}

Radiotelemetric units including transmitters with built in sensors (Data Sciences International, USA, Type: TL11M2-C50-PXT) were surgically implanted into rats as described before (Raffai et al. 2005, 2006). In brief, rats were anesthetized with sodium-pentobarbital (Sigma, USA, $50 \mathrm{mg} / \mathrm{kg} \mathrm{bw}$, ip), followed by a midline laparotomy. The non-occluding catheter of the pressure transducer was introduced into the abdominal aorta and fixed with a tissue adhesive. Bipolar ECG leads were tunneled subcutaneously into Lead II position, and fixed with surgical sutures.

\section{Data recording and signal analysis}

Body weight, food and water consumptions were measured daily throughout the experiment. Following a 10-day recovery period, body temperature $(\mathrm{T})$, and somatomotor activity (SA) signals sampled by $500 \mathrm{~Hz}$ and collected by RPC-1 receivers were fed into a Data Exchange Matrix and displayed on a PC. The signals were recorded and stored using Dataquest A.R.T. data acquisition system (Data Sciences International, USA).

Continuous blood pressure recording was made to determine diastolic blood pressure (DBP) systolic blood pressure (SBP), mean arterial blood pressure (BP), and heart rate (HR) in every $5 \mathrm{~s}$. Spontaneous baroreflex sensitivity (sBRS), as well as sympathetic and parasympathetic balance were calculated from 10-min segments of the continuous blood pressure recordings. Estimation of sBRS was made from spontaneous fluctuations in BP and HR using a time series method validated for rats (Oosting et al. 1997). In brief, 1) beatto-beat values of BP and pulse intervals were low-pass filtered using a 10-beat moving-average function, 2) the filtered signal was searched for ramps (decreasing or increasing BP) of four beats or more, and 3) the ratio of the BP ramp and pulse interval change at delays of 5 beats was determined, which served as a measure of 
sBRS. Power density spectral analysis of heart rate variability was performed on the pulse interval time series in order to estimate sympathetic and parasympathetic balance. Data sets of 512 points were resampled at $100 \mathrm{~ms}$, then Hamming window and fast Fourier transformation were applied to each set of data to obtain the power spectrum of the fluctuation. The power spectrum was divided into low frequency (LF, 0.04-1 Hz) and high frequency (HF, 1-3 Hz) ranges. Power of the LF, HF, and LF/HF was applied to estimate sympathetic and parasympathetic tone (Kuwahara et al. 1994).

\section{Experimental protocol}

Preceding the tilt test, $24 \mathrm{~h}$ recording was made with rats kept in traditional (normal) cages (NC). On the next day, the rats were placed into transparent tiltable tubular cages set in horizontal position (HOR). In this position, the rats were allowed to perform longitudinal locomotion along the whole length $(60 \mathrm{~cm})$ of the tubular cage, but they were not able to turn back. Chow and tap water were available at the edge of the cages. In the subsequent 7 days rats were kept in $45^{\circ}$ HDT position (HDT1-7). This experimental approach, which is regarded as a ground-based model of microgravity, can approximate the space flight conditions better than tail suspension model(s) (Monos et al. 2007, Lóránt et al. 2005).

Both preceding and following the 7-day HDT period, head-up tilt (HUT) tests were performed on the rats. Cages with the rats were set into HOR for $40 \mathrm{~min}$ and the last $10 \mathrm{~min}$ of this period was recorded which served as a control for the consecutive HUT for $2 \mathrm{~h}$.

Hydrostatic component of blood pressure responses in head-down tilt position

The distance between the cardiac zero reference level and the tip of the arterial catheter in the abdominal aorta was approximately $7 \mathrm{~cm}$. Consequently, HDT and HUT introduced a constant hydrostatic BP decrement and increment $(3.8 \mathrm{mmHg})$ between the heart and the catheter tip, respectively.

\section{Data evaluation and statistical analysis}

All measured and calculated variables were pooled for each hour, and these data points were used for the statistical evaluation. When circadian patterns of the physiological variables were studied, daylight and nighttime periods were pooled individually and expressed as daylight and nighttime values, respectively. In the case
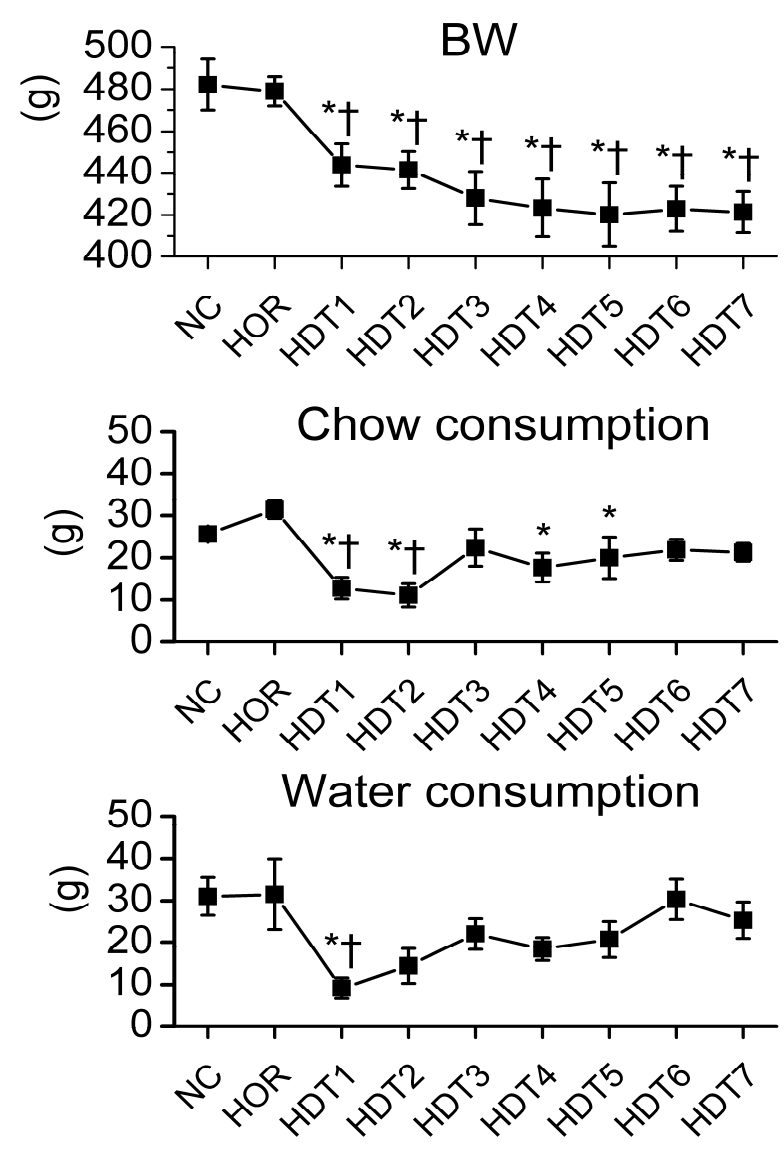

Fig. 1. Daily body weight (BW), chow and $\mathrm{H}_{2} \mathrm{O}$ consumption during the head-down tilt experiments. Variables were measured daily in traditional cages (NC), in confined horizontal position (HOR), and in head-down tilt position for 7 days (HDT1-7). Data are given as mean \pm S.E.M. $(n=8)$. Statistical differences $(\mathrm{P}<0.05)$ from $\mathrm{NC}$ and $\mathrm{HOR}$ values are indicated by $*$ and $\dagger$, respectively.

of HUT tests, a 10 min time resolution was applied. Results are given as means \pm S.E.M.. One-way repeated measures ANOVA was used for statistical analysis of data. Dunnett's pair-wise multiple comparison procedure was applied to test for significant differences from NC and HOR control data. A paired t-test was applied when daylight and nighttime values were compared. $\mathrm{P}<0.05$ is considered as statistically significant.

\section{Results}

Effect of 7 day HDT on body weight, food and water consumptions, body temperature and locomotor activity

During HDT average daily food and water intake of the rats transiently dropped, while body weight progressively decreased relative to both $\mathrm{NC}$ and $\mathrm{HOR}$ (Fig. 1). 


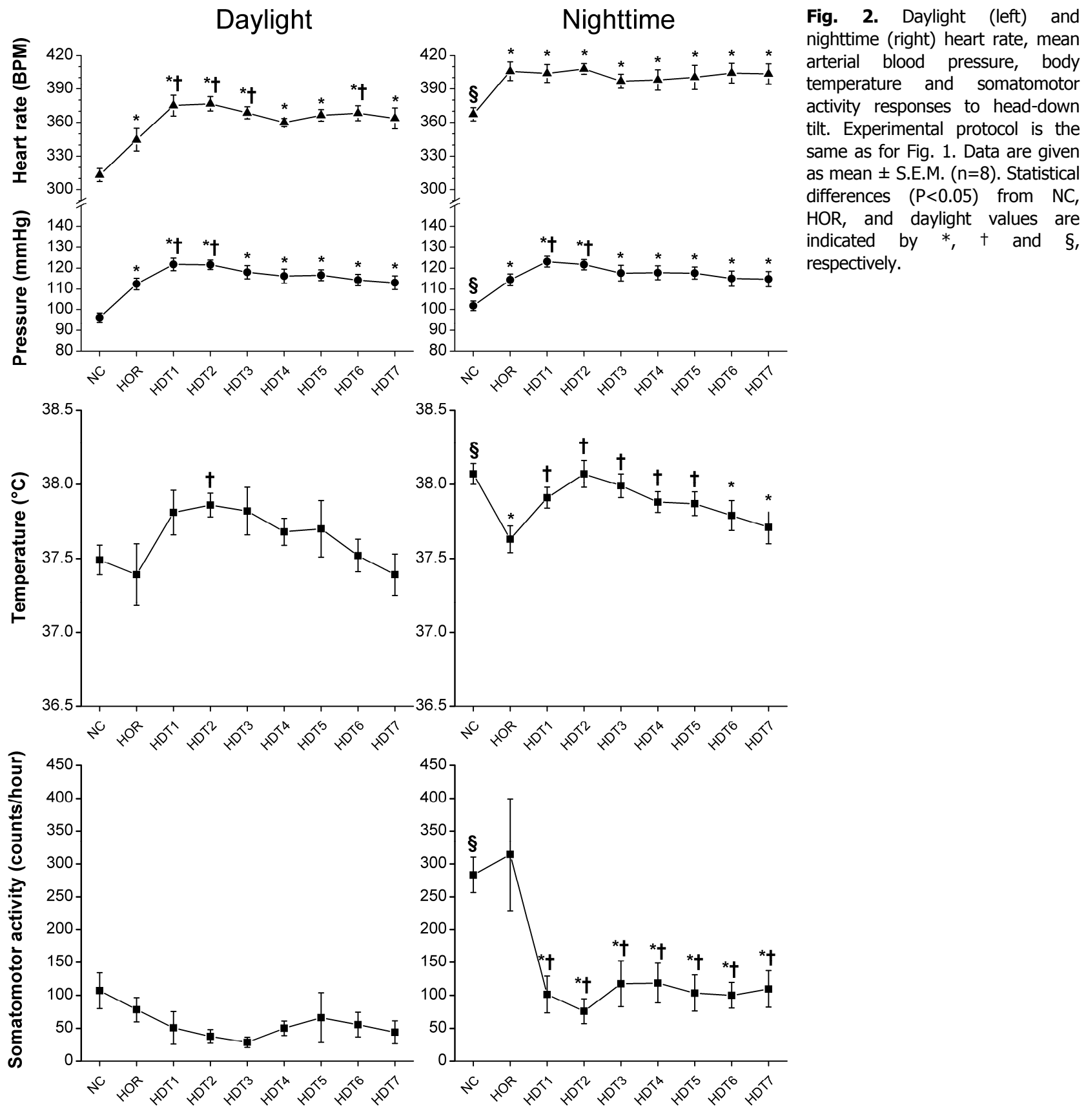

Nighttime temperature ( $\mathrm{T}$ ) and somatomotor activity (SA) were significantly higher $\left(38.1 \pm 0.1{ }^{\circ} \mathrm{C}\right.$, $283.3 \pm 26.8$ counts $/ \mathrm{h}$ ) compared to their respective daylight values $\left(37.5 \pm 0.1{ }^{\circ} \mathrm{C}, \quad 107.1 \pm 27.2\right.$ counts $/ \mathrm{h}$, $\mathrm{p}<0.05$ ) in traditional cages (Fig. 2, right and left, NC). HOR reduced nighttime $\mathrm{T}\left(37.6 \pm 0.1{ }^{\circ} \mathrm{C}, \mathrm{p}<0.05\right)$ while SA (314.2 \pm 85.3 counts/h) remained unchanged (Fig. 2, right, HOR). Nighttime $\mathrm{T}$ returned to its $\mathrm{NC}$ value in response to HDT (Fig. 2, right). Nighttime SA was reduced by approximately $60 \%$ from both NC and HOR during HDT ( $<<0.05$, Fig. 2, right, HDT1-7).
Changes of arterial blood pressure, heart rate, autonomic nervous activity and baroreflex sensitivity during 7-day HDT

Normal control values of basic cardiovascular parameters measured in traditional cages showed regular diurnal fluctuations. Average daylight/nighttime values of BP, DBP, SBP, and HR were $96.1 \pm 2.2 / 101.8 \pm 2.4 \mathrm{mmHg}$, $85.7 \pm 2.7 / 91.2 \pm 2.5 \mathrm{mmHg}, 108.4 \pm 2.9 / 114.4 \pm 3.6 \mathrm{mmHg}$, and $313.2 \pm 5.9 / 367.4 \pm 5.9 \mathrm{bpm}$, respectively $(\mathrm{p}<0.05$ for all parameters between daylight and nighttime, Fig. 2, left and right, NC). Neither calculated sBRS $(0.96 \pm 0.08 / 0.92 \pm 0.09 \mathrm{~ms} / \mathrm{mmHg})$ nor spectral properties 

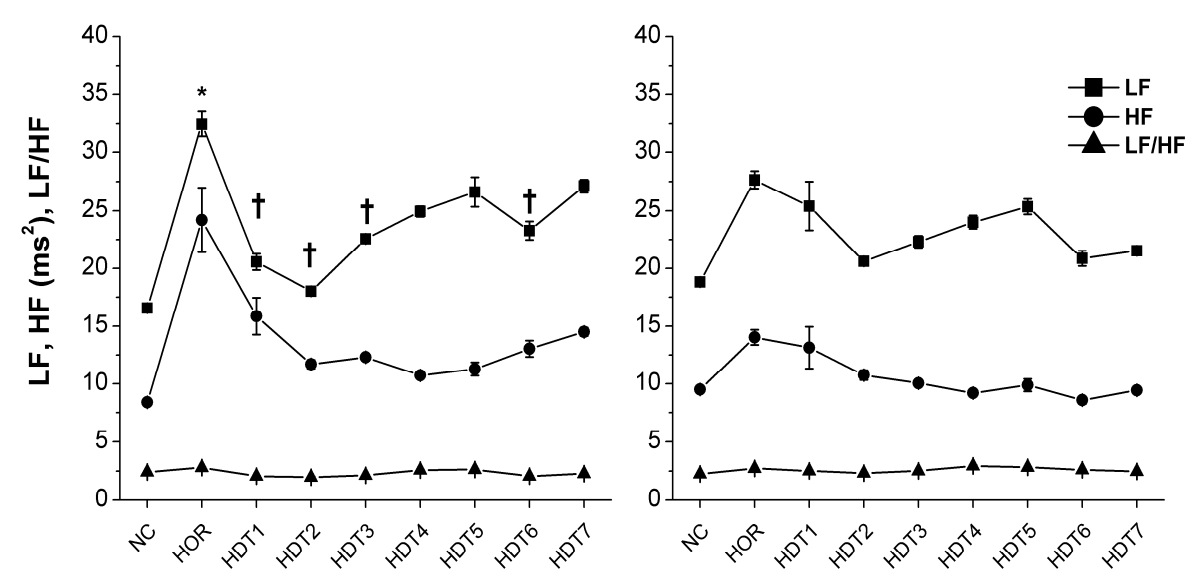

Fig. 3. Daylight (left) and nighttime (right) power of low-frequency (LF), high frequency (HF) components of the power spectrum of heart rate variability, their ratio (LF/HF), and spontaneous baroreflex sensitivity (sBRS) responses to head-down tilt. Experimental protocol is the same as for Fig. 1. Data are given as mean \pm S.E.M. $(n=5)$. Statistical differences $(P<0.05)$ from NC and HOR values are indicated by $*$ and $\dagger$, respectively.
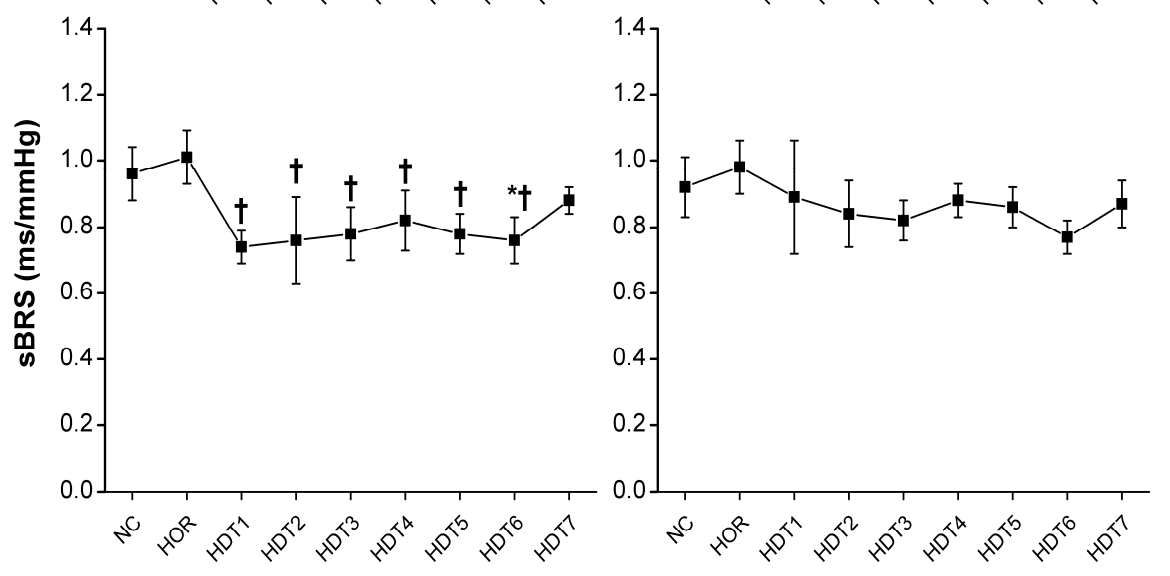

of continuous blood pressure recordings (LF: $16.6 \pm 0.3 / 18.8 \pm 0.3 \mathrm{~ms}^{2}$, HF: $8.4 \pm 0.2 / 9.5 \pm 0.2 \mathrm{~ms}^{2}, \mathrm{LF} / \mathrm{HF}$ : $2.4 \pm 0.04 / 2.3 \pm 0.06)$ showed circadian variations under control conditions (Fig. 3, NC).

Translocation of the rats from traditional cages to the horizontally positioned tubular cages had a significant impact on cardiovascular indexes. The confined horizontal position allowing free longitudinal motion, resulted in significant increases in both average daylight and nighttime values (daylight/nighttime) of BP $(112.5 \pm 2.8 / 114.5 \pm 2.8 \mathrm{mmHg})$, DBP $(97.6 \pm 3.3 / 101.9 \pm 3.8$ $\mathrm{mmHg})$, SBP $(134.4 \pm 6.6 / 129.8 \pm 3.2 \mathrm{mmHg})$, and HR $(344.7 \pm 10.1 / 405.9 \pm 8.5 \mathrm{bpm}),(\mathrm{p}<0.05$ for all parameters, Fig. 2, left and right, HOR). These changes were accompanied by an increase of LF in the daylight (32.4 $\pm 1.1 \mathrm{~ms}^{2}$, Fig. 3, left, HOR). Other calculated parameters were not influenced by HOR.

Head-down tilt induced a further elevation of cardiovascular variables compared with the confined horizontal position. Thus, daylight BP, DBP, and HR increased by $9.4 \mathrm{mmHg}, 12 \mathrm{mmHg}$, and $31 \mathrm{bpm}<1 \mathrm{~h}$ $(\mathrm{p}<0.05)$, for 2,3 and 3 days, respectively, with a subsequent normalization at the HOR level (Fig. 2, left, HDT1-7). Nighttime values of BP, DBP, and SBP increased similarly, but HR did not change (Fig. 2, right, HDT1-7). Daylight LF returned to its NC value as an effect of HDT (Fig. 3, right). In comparison to HOR, HDT resulted in a daylight reduction of baroreflex sensitivity by $0.13-0.28 \mathrm{~ms} / \mathrm{mmHg}$ (Fig. 3, left).

Effect of 2-h HUT on cardiovascular responses before and after 7-day HDT

Two hours of HUT, performed before chronic HDT, resulted in increased mean arterial pressure (by 5.0-13.7 $\mathrm{mmHg}$ ), increased diastolic pressure (by 4.8$13.9 \mathrm{mmHg}$ ), and increased systolic pressure (by 5.7-14.1 $\mathrm{mmHg}$ ) compared to their respective HOR values $(\mathrm{p}<0.05$, Fig. 4a). HUT elicited a decrease in sBRS (by $0.28-0.54 \mathrm{~ms} / \mathrm{mmHg}$ ) during the 2-h HUT observation period (Fig. 5a).

Following 7-day HDT, cardiovascular parameters measured in the confined horizontal position returned to their pre-HDT HOR levels within one hour (Figs 2, 3, 4, and 5). The post-HDT HUT test (lasting for $2 \mathrm{~h}$ ) also resulted in increased mean arterial pressure (by 10.7-16.4 mmHg), diastolic pressure (by 9.3-16.4 $\mathrm{mmHg}$ ), and systolic pressure (by 10-15.6 $\mathrm{mmHg}$ ) compared to the HOR values $((p<0.05$, Fig. $4 b)$. In contrast to the pre-HDT HUT tests, the pressor response in the post-HDT HUT test was accompanied by an increase in HR (by 35.5-66.8 bpm). Similar to the preHDT test, sBRS decreased (by 0.16-0.48 $\mathrm{ms} / \mathrm{mmHg}$ ) 

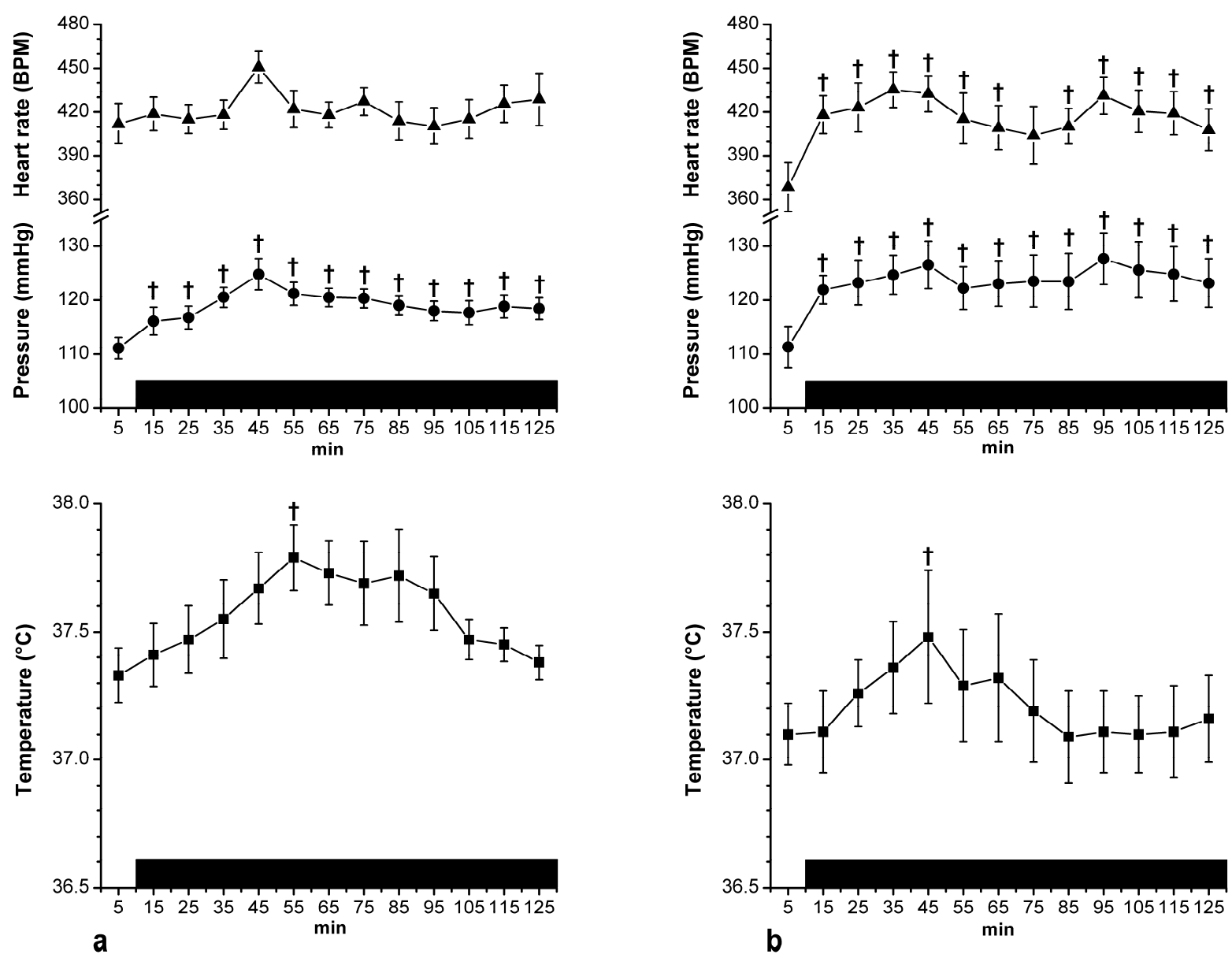

Fig. 4. Responses of heart rate, mean arterial blood pressure, and body temperature to head-up tilt a) before and b) after chronic HDT. Experimental protocol as follows: 0-10 minutes horizontal position (not labeled), 10-130 minutes head-up tilt (filled band). Data are given as mean \pm S.E.M. $(n=8)$. Statistical difference $(P<0.05)$ from HOR is indicated by + .

during the 2-h post-HDT HUT observation period (Fig. 5b).

Neither $\mathrm{T}$ nor spectral properties (LF, HF, $\mathrm{LF} / \mathrm{HF}$ ) were influenced by either of the HUT tests (Figs 4 and 5).

\section{Discussion}

The present study made three major new observations. First, behavioral patterns were influenced by chronic antiorthostasis as evidenced by suppression of nighttime somatomotor and the feeding activities. Second, 7-day HDT increased arterial blood pressure and heart rate for 2 to 3 days and led to a significant reduction in daylight baroreflex sensitivity. Finally pre- and postHDT orthostasis tests resulted in a sustained rise of arterial blood pressure and an impaired baroreflex sensitivity. During the latter challenge (post-HDT), blood pressure rise tended to be increased, which was accompanied with a tachycardic response.

\section{Behavioral responses}

Behavioral patterns were influenced specifically by HDT. Namely, the HDT produced a decrease in nighttime locomotor activity (compared to $\mathrm{NC}$ and HOR). There is evidence, that stressful conditions can lead to "freezing" reactions (Inagaki et al. 2004), which could be a possible explanation to our observation. In our case, unlike HDT, a change in the microenvironment (traditional vs. tubular cage in HOR position) was not accompanied by reduction of somatomotor activity, even though the rats were confined to uniaxial locomotion. Therefore, instead of freezing reactions, we propose the explanation that the rats spend most of their time to maintain their position against gravitation which increases their physical performance. Similar to the case with tail suspension models (Musacchia and Fagette 1997) the hypokinesia observed in the present experiment seems to be attributable to the HDT body position. A progressive decrease in body weight, while feeding activity (chow and water consumption) returns to normal 

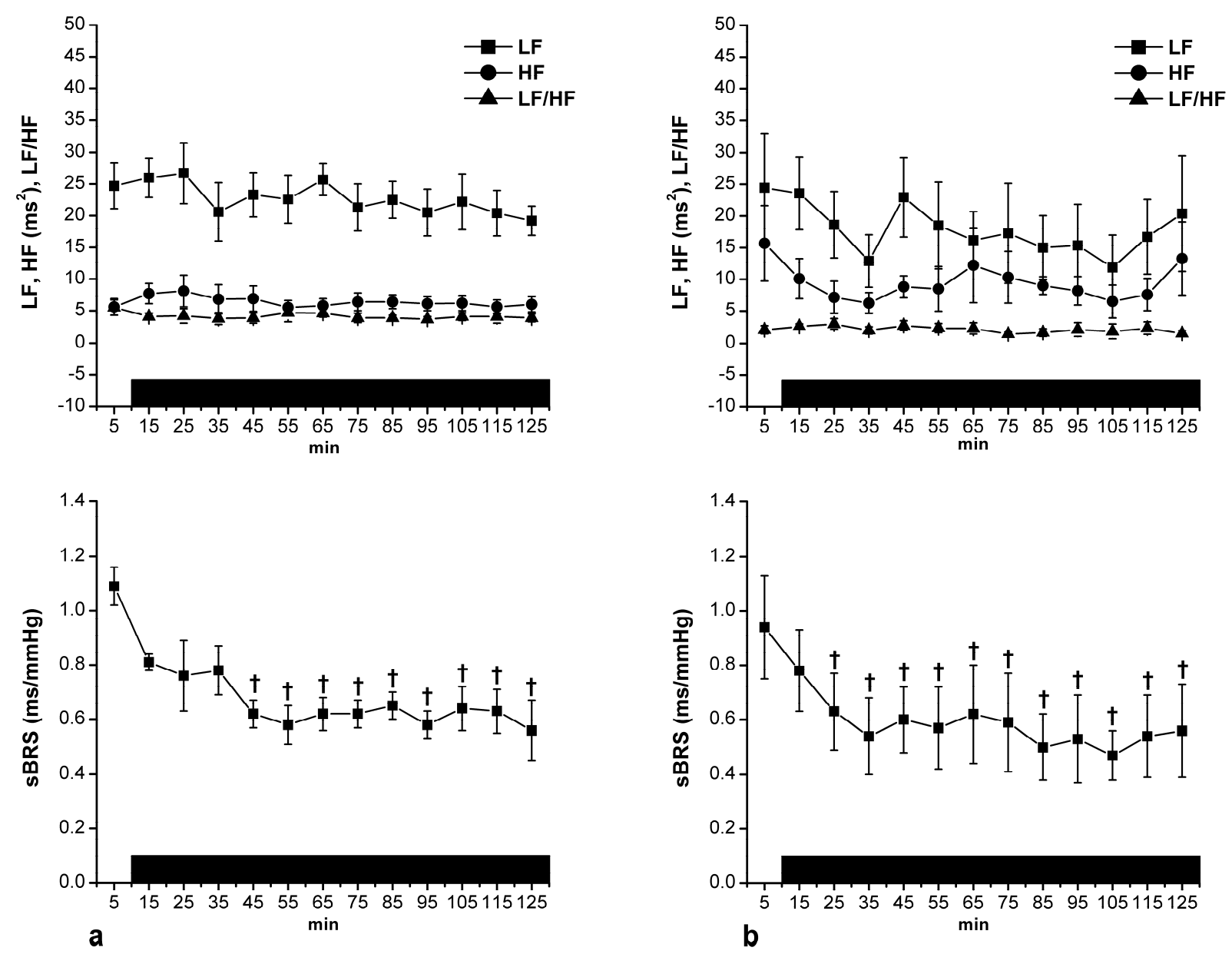

Fig. 5. Responses of low-frequency (LF) and high frequency (HF) components of the power spectrum of heart rate variability, their ratio (LF/HF), and spontaneous baroreflex sensitivity (sBRS) to head-up tilt a) before $(n=7)$ and b) after $(n=5)$ chronic HDT. Experimental protocol as follows: 0-10 minutes horizontal position (not labeled), 10-130 minutes head-up tilt (filled band). Data are given as mean \pm S.E.M. Statistical difference $(P<0.05)$ from HOR is indicated by + .

after an initial drop supports this theory. The absence of a substantial non-specific, non-gravitational stress was also confirmed by the lack of hyperthermia that is usually caused by immobilization stress (Saiki et al. 1997), as well as by the unexpected behavior of the rats who tend to accept tubular cages as a familiar surrounding throughout the study.

Effect of non-specific and non-gravitational stress on baseline cardiovascular parameters

In the present study, average BP values were quite similar to those of Musacchia's presuspension controls (Musacchia et al. 1992), but HR was substantially lower in NC. These discrepancies found between control HR values in our and Musacchia's studies are probably not due to the difference between rat strains (Sprague-Dawley vs. Wistar) (McDougall et al. 2005), but most likely represent differences in experimental protocols (e.g. chronic catheterization or suspension harness). The latter represents a substantial stressful condition, which could have a more pronounced effect on HR than on BP in non-telemetered rats (Irvine et al. 1997), and can subsequently change the physiological status of the animals (Irvine et al. 1997).

Restraint stress and mild handling procedures can elicit transient cardiovascular responses in rats (Irvine et al. 1997, McDougall et al. 2000, 2005, Raffai et al. 2005, 2006) with full (Irvine et al. 1997) or partial restoration of BP (McDougall et al. 2000, 2005, Raffai et al. 2005, 2006). In our study, transferring the rats from traditional cages to tubular tilt cages caused a sustained increase in BP and HR, which remained somewhat above $\mathrm{NC}$ as it was seen in our previous studies (Raffai et al. 2005, 2006). HOR BP values were also comparable to those of the non-HDT rats equipped with the suspension harness (Musacchia et al. 1992), while HOR HR values still remained below it, with the only exception in the preHDT orthostasis test. In this latter case the relatively 
elevated HR is attributed to an augmented rise of $\mathrm{HR}$ $(+160 \mathrm{BPM})$ when transferring the rats from $\mathrm{NC}$ to HOR, followed by a similar restoration (-66 BPM) as it was seen previously (Raffai et al. 2005, 2006). In either of the cases, such a maintained rise of cardiovascular variables in confined horizontal position indicates the presence of a constant level of non-specific stress that could be eliminated pharmacologically (Raffai et al. 2005). Moreover, such a partial restoration of the cardiovascular parameters still provided an adequate reserve for further BP or HR responses induced by either HUT or HDT superimposed on HOR values.

Compared to 24-h HOR and post-HDT HOR values, there was a marked depression of $\mathrm{HF}$ and an increase of LF/HF in HOR of pre-HDT orthostasis test, suggesting a decreased parasympathetic cardiac control. This observation agrees well with the observed elevated HR, but the indicated biobehavioral changes (Kuwahara et al. 1994) were not adequate either to prevent hypertensive BP response or to alter LF during the subsequent pre-HDT orthostasis test.

\section{Cardiovascular responses to HDT}

During the first two days of HDT, rats responded with BP elevations in both daylight $(10 \%)$ and nighttime (7-9 \%) periods. Unlike BP responses, only daylight rise of HR was seen till the third day of HDT (8-10\%). None of the spectral parameters changed, while spontaneous baroreflex sensitivity was reduced throughout HDT. Our results during chronic HDT in the tubular tilt cage agree well with those of Musacchia et al. (1992) who measured BP and HR increases in Sprague-Dawley rats from the first day using the tail suspension model. However, these results are in contrast with those of others, who also used the tail suspension model but observed no change either in BP (Somody et al. 1998, Tarasova et al. 2001) or in both BP and HR (Brizzee and Walker 1990, Fagette et al. 1995, Bayorh et al. 1999, Socci et al. 2000, Bayorh et al. 2001, Eatman et al. 2003).

The characteristics of dual circulatory responses (changes in both BP and HR) suggest that both cardiovascular reflexes and neuroendocrine mechanisms are involved in adaptation to HDT. Antiorthostasis presumably initiates increases in sympathetic neural output and circulating catecholamines, as well as other vasoconstrictor hormones that elevate both BP and HR. This conclusion is in accordance with our earlier results, that BP rise due to 2-h HDT was prevented by sympathetic blockade (Raffai et al. 2006). One of the causes of the increased sympathetic discharge is probably the cephalad fluid shift(s) that at least doubles intracerebroventricular pressure within 10 min (Assenmacher et al. 1995, Maurel et al. 1996), and may induce neuroendocrine and neurophysiological disorders in rats (Assenmacher et al. 1995). Central angiotensin II receptors are likely to be involved in antiorthostatic responses, as well as other stress-induced increases of sympathetic nervous and adrenomedullary activities that are accompanied by pressor response and tachycardia (Saiki et al. 1997).

Gravity-related reflexes due to changes in body position can also be activated by several receptor mechanisms in addition to the well-known baroreceptor system. Labyrinthine, proprioceptive, visual, visceral (e.g. cardiopulmonary mechanoreceptors), and cutaneous inputs, all of which converge on the neurons of the central vestibular nuclei, have also been implicated in cardiovascular responses (Morita et al. 2000, Yates et al. 2000) These inputs are then transmitted to the circulatory control area of the brainstem influencing sympathetic output. This in turn, increases precapillary vascular resistance, venous tone, arterial BP and HR (Yates et al. 2000), compensating for the imbalance caused by cephalad fluid shift(s) and redistribution of the blood. The operation and physiological significance of vestibulosympathetic reflexes are also well supported by experimental evidence in both humans and animals (Monos and Lóránt 1998, Yates et al. 2000, Raffai et al. unpublished observations). Thus, it is reasonable to assume that long-term HDT may induce a significant rise in BP and HR via such neural reflexes. In contrast to acute $(2$ h) HDT (Raffai et al. 2006), reduced sBRS during chronic HDT - lasting one week (Brizzee and Walker 1990) or two weeks (Fagette et al. 1995) - is either a consequence of postural change or it is an impairment of HR decreasing mechanism. Elevated HR, which was absent in short-term HDT experiments (Raffai et al. 2006), may be attributed to the reduction of sBRS which would promote BP rise in HDT. This assumption can be explained either by the modulation of baroreflex function or by a readaptation of central and peripheral cardiovascular regulatory mechanisms (Morita et al. 2000) elicited by cephalad fluid shifts and increase in transmural pressure (Morita et al. 2000). In the present study, autonomic balance was preserved because neither of the spectral properties (LF, HF, LF/HF) (Kuwahara et al. 1994) changed in parallel with HDT. This observation is in good agreement with the results obtained during two 
weeks of tail suspension (Fagette et al. 1995). In the present study, rise in the HR suggests some degree of sympathetic activation during the first 3 days of the HDT. In our case, a small sympathetic activation or a small parasympathetic withdrawal occurring at a high parasympathetic tone, could cause substantial elevation of HR that would not essentially be reflected by the spectral parameters. Moreover, tachycardia in the present experiments was accompanied by a pressor response that could activate reflexes, limiting the increase in HR. Our hypothesis concerning the autonomic state during exposure to long-term antiorthostatic stress is supported by others who reported that autonomic balance predominates in anxiety-like and fear-like states (Inagaki et al. 2004), and that BP induced reflex responses can oppose other neural influences that control HR (McDougall et al. 2000).

\section{Lack of deconditioning - disturbance in post-HDT cardiovascular responses}

The one-week time course of chronic HDT test in this study was chosen because 7 days appears to be sufficient to elicit cardiovascular deconditioning in microgravity models (Socci et al. 2000, Bayorh et al. 2001, Eatman et al. 2003). In another study, a decreased pressor response was attributed to cardiovascular decondititoning from the third day of tail suspension (Musacchia et al. 1992). After the one-week period of HDT in the present experiments, the rats did not show any symptom of cardiovascular deconditioning, as all post-HDT HOR cardiovascular parameters returned to their pre-HDT tilt level within an hour. This agrees well with the results of investigators who saw a full return or no change in cardiovascular parameters (Brizzee and Walker 1990, Musacchia et al. 1992, Fagette et al. 1995, Somody et al. 1998, Bayorh et al. 1999, Bayorh et al. 2001, Tarasova et al. 2001), but contrasts with studies reporting a reduction of BP (Bayorh et al. 2001, Eatman et al. 2003, Socci et al. 2000) after tail suspension.

Similarly to the study of Musacchia et al. (1992), we applied pre- and post-HDT HUT tests (2 h), in order to assess the degree of a possible cardiovascular deconditioning. During these periods of orthostasis testing, the rats responded equally with elevated blood pressure and a reduction of baroreflex sensitivity. While the post-HDT hypertensive response tended to be increased, neither pre- nor post-HDT BP and sBRS responses were qualitatively or quantitatively different. In contrast to pre-HDT or to orthostatic responses observed earlier (Raffai et al. 2005), the 2-h post-HDT orthostasis test elicited a maintained tachycardic response, that might contribute to the augmented BP rise.

The absence of post-HDT cardiovascular deconditioning (indicated by a failure to maintain arterial blood pressure) in our microgravity model remains unknown. Position-dependent reduction of sBRS cannot be simply explained by direct hemodynamic changes, because opposite changes would have occurred in the HDT and in HUT positions. We propose that, impaired baroreflex sensitivity in the orthostatic position can contribute to the elevated HR during post-HDT orthostasis testing. However, such a reduction of sBRS is insufficient to cause cardiovascular deconditioning, but the accompanying HR elevation might reflect an initial phase of it. Moreover, other proprioceptive inputs (Yates et al. 2000) can compensate for the inadequate baroreflex operation and the increase in HR. For example, our preliminary data show that the vestibular system can play a significant role in orthostatic BP and HR responses (Raffai et al. unpublished observation). In the latter case, there could well be a cooperative contribution of the baroreflex and the vestibulosympathetic reflex in maintaining arterial blood pressure during gravitational stress and postural changes (Gotoh et al. 2004).

\section{Conclusions}

It can be concluded, that an imbalance caused by chronic antiorthostatic challenge activates complex cardiovascular compensatory mechanisms until a new steady state is reached. In both the antiorthostatic (HDT) and orthostatic (HUT) positions, the deleterious effect of an impaired baroreflex function is ameliorated by other sensory inputs (e.g. labyrinthine, proprioceptive, visual, visceral, cutaneous etc.) via the corresponding reflexes to prevent cardiovascular deconditioning and to maintain arterial blood pressure.

\section{Conflict of Interest}

There is no conflict of interest.

\section{Acknowledgements}

This work was supported by OTKA T-042670/2003 and ETT 128/2006 Hungarian Grants. We are grateful to Dr. Julian H. Lombard for his help in editing the manuscript. Authors are indebted to Drs. Sándor Farkas and Márk Kollai for their continuous scientific support. 


\section{References}

ASSENMACHER I, MEKAOUCHE M, MAUREL D, BARBANEL G, GIVALOIS L, BOISSIN J, MALAVAL F, IXART G: Chronic orthostatic and antiorthostatic restraint induce neuroendocrine, immune and neurophysiologial disorders in rats. Acta Astronautica 36: 545-558, 1995.

BAYORH MA, SOCCI RR, WANG M, THIERRY-PALMER M, EMMETT N: Influence of simulated microgravity on cardiovascular and hemodynamic parameters in Dahl salt-sensitive rats. J Gravit Physiol 6: 63-70, 1999.

BAYORH MA, EATMAN D, WANG M, SOCCI RR, EMMETT N, THIERRY-PALMER M: Indomethacin attenuates post-suspension hypotension in Sprague-Dawley rats. J Gravit Physiol 8: 77-83, 2001.

BRIZZEE BL, WALKER BR: Altered baroreflex function after tail suspension in the conscious rat. $J$ Appl Physiol 69: 2091-2096, 1990.

BROCKWAY BP, MILLS PA, AZAR SH: A new method for continuous chronic measurement and recording of blood pressure, heart rate and activity in the rat via radio-telemetry. Clin Exp Hypertens 13: 885-895, 1991.

CONVERTINO VA: G-factor as a tool in basic research: mechanisms of orthostatic tolerance. J Gravit Physiol 6: 73$76,1999$.

CONVERTINO VA: Mechanisms of microgravity induced orthostatic intolerance: implications for effective countermeasures. J Gravit Physiol 9: 1-13 2002a.

CONVERTINO VA: Planning strategies for development of effective exercise and nutrition countermeasures for longduration space flight. Nutrition 18: 880-888, 2002b.

EATMAN D, WALTON M, SOCCI RR, EMMETT N, BAYORH MA: NOS II inhibition attenuates post-suspension hypotension in Sprague-Dawley rats. Clin Exp Hypertens 25: 11-24, 2003.

FAGETTE S, LO M, GHARIB C, GAUQUELIN G: Cardiovascular variability and baroreceptor reflex sensitivity over a 14-day tail suspension in rats. $J$ Appl Physiol 78: 717-724, 1995.

GOTOH TM, FUJIKI N, MATSUDA T, GAO S, MORITA H: Roles of baroreflex and vestibulosympathetic reflex in controlling arterial blood pressure during gravitational stress in conscious rats. Am J Physiol 286: R25-R30, 2004.

INAGAKI H, KUWAHARA M, TSUBONE H: Effects of psychological stress on autonomic control of heart in rats. Exp Anim 53: 373-378, 2004.

IRVINE RJ, WHITE J, CHAN R, DAI SH: The influence of restraint on blood pressure in the rat. $J$ Pharmacol Toxicol Methods 38: 157-162, 1997.

KUWAHARA M, YAYOU K, ISHII K, HASHIMOTO S, TSUBONE H, SUGANO S: Power spectral analysis of heart rate variability as a new method for assessing autonomic activity in the rat. $J$ Electrocardiol 27: 333-337, 1994.

LÓRÁNT M, RAFFAI G, NÁDASY GL, FEHÉR E, MONOS E: Does chronic experimental head-down tilt alter intramural innervation density of limb blood vessels? Jpn J Physiol 55: 127-134, 2005.

MAUREL D, IXART G, BARBANEL G, MEKAOUCHE M, ASSENMACHER I: Effects of acute tilt from orthostatic to head-down antiorthostatic restraint and of sustained restraint on the intracerebroventricular pressure in rats. Brain Res 736: 165-173, 1996.

MCDOUGALL SJ, PAULL JR, WIDDOP RE, LAWRENCE AJ: Restraint stress: differential cardiovascular responses in Wistar-Kyoto and spontaneously hypertensive rats. Hypertension 35: 126-129, 2000.

MCDOUGALL SJ, LAWRENCE AJ, WIDDOP RE: Differential cardiovascular responses to stressors in hypertensive and normotensive rats. Exp Physiol 90: 141-150, 2005.

MONOS E, LÓRÁNT M: Vestibular control of the cardiovascular system. Orv Hetil 139: 1851-1855, 1998.

MONOS E, RAFFAI G, DÖRNYEI G, NÁDASY GL, FEHÉR E: Structural and functional responses of extremity veins to long-term gravitational loading or unloading - lessons from animal systems. Acta Astronautica 60: 406-414, 2007.

MORITA H, FUJIKI N, TSUCHIYA Y, MIYAHARA T, TANAKA K: Afferent mechanisms of acute responses of renal sympathetic nerve activity to microgravity. J Gravit Physiol 173-174, 2000.

MUSACCHIA XJ, FAGETTE S: Weightlessness simulations for cardiovascular and muscle systems: validity of rat models. J Gravit Physiol 4: 49-59, 1997. 
MUSACCHIA XJ, STEFFEN JM, DOMBROWSKI J: Rat cardiovascular responses to whole body suspension: headdown and non-head-down tilt. J Appl Physiol 73: 1504-1509, 1992.

OOSTING J, STRUIJKER-BOUDIER HA, JANSSEN BJ: Validation of a continuous baroreceptor reflex sensitivity index calculated from spontaneous fluctuations of blood pressure and pulse interval in rats. J Hypertens $\mathbf{1 5}$ : 391-399, 1997.

RAFFAI G, MÉSZÁROS M, KOLLAI M, MONOS E, DÉZSI L: Experimental orthostasis elicits sustained hypertension, which can be prevented by sympathetic blockade in the rat. J Cardiovasc Pharmacol 45: $354-$ 361, 2005.

RAFFAI G, KOCSIS L, MÉSZÁROS M, MONOS E, DÉZSI L: Inverse-orthostasis may induce elevation of blood pressure due to sympathetic activation. J Cardiovasc Pharmacol 47: 287-294, 2006.

SAIKI Y, WATANABE T, TAN N, MATSUZAKI M, NAKAMURA S: Role of central ANG II receptors in stressinduced cardiovascular and hyperthermic responses in rats. Am J Physiol 272: R26-R33, 1997.

SOCCI RR, WANG M, THIERRY-PALMER M, EMMETT N, BAYORH MA: Cardiovascular responses to simulated microgravity in Sprague-Dawley rats. Clin Exp Hypertens 22: 155-164, 2000.

SOMODY L, FAGETTE S, BLANC S, FRUTOSO J, GHARIB C, GAUQUELIN-KOCH G: Regional blood flow in conscious rats after head-down suspension. Eur J Appl Physiol Occup Physiol 78: 296-302 1998.

TARASOVA O, FIGOURINA I, ZOTOV A, BOROVIK A, VINOGRADOVA O: Effect of tail suspension on haemodynamics in intact and sympathectomized rats. Eur J Appl Physiol 85: 397-404, 2001.

YATES BJ, HOLMES MJ, JIAN BJ: Adaptive plasticity in vestibular influences on cardiovascular control. Brain Res Bull 53: 3-9, 2000.

ZHANG LF: Vascular adaptation to microgravity: what have we learned? J Appl Physiol 91: 2415-2430, 2001.

ZHANG LF, YU ZB, MA J, MAO QW: Peripheral effector mechanism hypothesis of postflight cardiovascular dysfunction. Aviat Space Environ Med 72: 567-575, 2001. 